\title{
Neues aus der Schweizerischen Gesellschaft für Gynäkologische Endokrinologie und Menopause/SGEM
}

J. Gynäkol. Endokrinol. CH $2021 \cdot 24: 103$

https://doi.org/10.1007/s41975-021-00203-1

๑) Springer-Verlag GmbH Austria, ein Teil von Springer Nature 2021

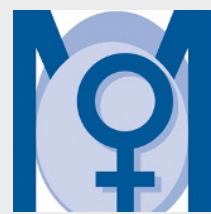

Präsidentin

Prof. Dr. med. Petra Stute (V.i. S. d. P.)

Administration SGEM

Weißdornweg 17

D-35041 Marburg/Lahn

Tel.: +49 (0) 642093444

E-Mail: administration@meno-pause.ch
Liebe Kolleginnen und Kollegen!

Immer noch Pandemie! Aufgrund der nach wie vor unsicheren Situation haben wir uns entschieden, den Women's Health Kongress vom 09.-10.06.2021 auf das nächste Jahr zu verschieben. Somit sehen wir uns alle $\mathrm{zu}$ gewohnter Jahreszeit wieder vom 02.-03.02.2022 findet der Women's Health Kongress in Aarau statt. Wir freuen uns schon sehr darauf!

\section{Blockkurs SGGG/SGEM, ONLINE}

Am 23.10.2021 findet der Blockkurs „Gynäkologische Endokrinologie und Reproduktionsmedi- zin" unter der wissenschaftlichen Leitung der SGEM mit folgenden Themen statt:

- Update hormonelle Kontrazeption

- ESHRE Guideline zum Management des PCOS

- Kinderwunschtherapie in der frauenärztlichen Praxis

- Knochengesundheit in der Peri- und Postmenopause

- Update Menopausale Hormontherapie

- Management von Blutungsstörungen

- Neue Techniken und Gesetzesgrundlagen in der Reproduktionsmedizin

Ebenfalls möchten wir Sie auf die Fortbildungen der Deutschen
Menopause Gesellschaft e. V. hinweisen:

\section{Frauen „Ü40" endokrinologisch verstehen... die Prä-, Peri- und Postmenopause}

- Curriculum Modul II „Was sollten Frauenärzte/Innen aus anderen Fachgebieten wissen?"

- 05.-06.11.2021, ONLINE

- Curriculum Modul I „Basiswissen Endokrinologie“

- 03.-04.12.2021, Hamburg oder ONLINE

Werden Sie Mitglied in der Schweizerischen Gesellschaft für Gynäkologische Endokrinologie und Menopause - SGEM

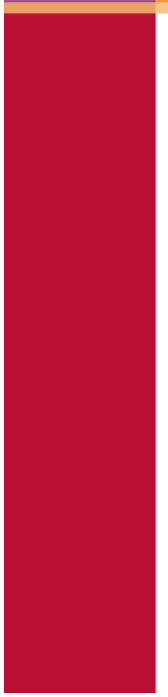

- Ermässigte Teilnahmegebühren: Women's Health Kongress

Curricula Menopause „Frauen Ü40", Jahrestagung der DMG e.V. und teilweise bei Tagungen anderer Fachgesellschaften

- Kostenloses Abonnement der Fachzeitschrift

„Journal für Gynäkologische Endokrinologie“" inkl. Online-Zugang

- Informationen zu aktuellen Richt- und Leitlinien - Zugang zu Vorträgen der Women's Health Kongresse

- Factsheets in D, F und I zu verschiedenen Themen der Menopause

- Beantwortung von Fachfragen durch den Vorstand Anmeldung unter: www.meno-pause.ch/aufnahmeantrag_mitgliedschaft

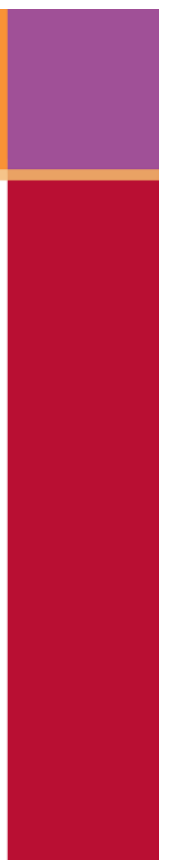

DMG Jahrestagung 2021

„Gesund von Kopf bis Fuß durch die Lebensmitte“

12.-13.11.2021, Frankfurt am Main oder Online-Kongress

Weitere Informationen unter: www.menopause-gesellschaft.de Und denken Sie daran, als SGEM Mitglied erhalten Sie die Vorteilspreise der DMG Mitglieder!

\section{SGEM Science Award 2022}

Einreichungen zum SGEM Science Award 2022 senden Sie bitte bis 01.12.2021 an administration@meno-pause.ch. Die Verleihung erfolgt während des Women's Health Kongress 2022 in Aarau. Details zur Ausschreibung finden Sie unter www.meno-pause.ch oder Sie wenden sich an unsere Administration. Wir freuen uns schon jetzt auf Ihre Einreichung!

Mit kollegialen Grüssen,

\section{Ihre Petra Stute}

Präsidentin Schweizerische Gesellschaft für Gynäkologische Endokrinologie und Menopause (SGEM)

\section{Kontaktadresse}

Administration, Anne Becker

Schweizerische Gesellschaft für Gynäkologische Endokrinologie und Menopause (SGEM)

Weißdornweg 17

D-35041 Marburg/Lahn

E-Mail: administration@

meno-pause.ch 